\title{
Evaluation of Five Antischizophrenic Agents Against Toxoplasma gondii in Human Cell Cultures
}

\author{
Author(s): David G. Goodwin , Jeannine S. Strobl , and David S. Lindsay \\ Source: Journal of Parasitology, 97(1):148-151. 2011. \\ Published By: American Society of Parasitologists \\ DOI: http://dx.doi.org/10.1645/GE-2536.1 \\ URL: http://www.bioone.org/doi/full/10.1645/GE-2536.1
}

BioOne (www.bioone.org) is a nonprofit, online aggregation of core research in the biological, ecological, and environmental sciences. BioOne provides a sustainable online platform for over 170 journals and books published by nonprofit societies, associations, museums, institutions, and presses.

Your use of this PDF, the BioOne Web site, and all posted and associated content indicates your acceptance of BioOne's Terms of Use, available at www.bioone.org/page/terms_of_use.

Usage of BioOne content is strictly limited to personal, educational, and non-commercial use. Commercial inquiries or rights and permissions requests should be directed to the individual publisher as copyright holder. 


\title{
Evaluation of Five Antischizophrenic Agents Against Toxoplasma gondii in Human Cell Cultures
}

\author{
David G. Goodwin, Jeannine S. Strobl', and David S. Lindsay†, Department of Biomedical Sciences and Pathobiology, Virginia-Maryland \\ Regional College of Veterinary Medicine, Virginia Tech, Blacksburg, Virginia 24061-0342; *Department of Electrical and Computer Engineering, \\ Virginia Tech, Blacksburg, Virginia 24061; †To whom correspondence should be addressed. e-mail:lindsayd@vt.edu
}

ABSTRACT: An increasing interest in the association of the presence of antibodies to Toxoplasma gondii and the development of schizophrenia in patients has been generated over the last several years. Some antischizophrenia agents have been shown to have activity against $T$. gondii in cell culture assays and to ameliorate behavioral changes associated with chronic $T$. gondii infection in rats. In the present study, we examined the effects of commonly used antipsychotic and mood stabilizing agents (haloperidol, clozapine, fluphenazine, trifluoperazine, and thioridazine) for activity against developing tachyzoites of the RH strain of $T$. gondii in human fibroblast cell cultures. Neither haloperidol nor clozapine had a measurable effect. Fluphenazine had an $\mathrm{IC}_{50}$ of $1.7 \mu \mathrm{M}$, thioridazine had an $\mathrm{IC}_{50}$ of $1.2 \mu \mathrm{M}$, and trifluoperazine had an $\mathrm{IC}_{50}$ of $3.8 \mu \mathrm{M}$. Our study demonstrates that some agents used to treat schizophrenia have the ability to inhibit $T$. gondii proliferation in cell culture.

It has been estimated that the prevalence of schizophrenia is approximately $1 \%$ in the American population (Regier et al., 1993) Several research groups from different countries have shown that there is an increase in antibodies against the zoonotic protozoan Toxoplasma gondii in individuals with schizophrenia compared to individuals without the disease in the same populations (Alvarado-Esquivel et al., 2006; Wang et al., 2006; Cetinkaya et al., 2007; Niebuhr et al., 2008). Researchers have also shown that children born to mothers with antibodies to $T$. gondii are at an increased risk of developing schizophrenia (Brown et al., 2005; Mortensen, Nørgaard-Pedersen, Waltoft, Sørensen, Hougaard et al., 2007; Mortensen, Nørgaard-Pedersen, Waltoft, Sørensen, Hougaard, Torrey, et al., 2007). One study demonstrated that patients with schizophrenia and positive for antibodies to $T$. gondii had a significantly increased risk of dying from natural causes compared to patients with schizophrenia and no antibodies to $T$. gondii (Dickerson et al., 2007).

It has recently been proposed that schizophrenia susceptibility genes are directly implicated in life cycle of $T$. gondii and other neuropathogens in the human brain (Carter, 2009). These observations suggest that there is a pathobiological relationship between $T$. gondii infection and the occurrence of schizophrenia. Researchers have demonstrated that some agents used to treat schizophrenia and other psychological disorders have activity against $T$. gondii in cell culture systems (Jones-Brando et al., 2003; Goodwin et al., 2008). In the present study, we examined the effects of commonly used antipsychotic and mood stabilizing agents (haloperidol, clozapine, fluphenazine, trifluoperazine, and thioridazine) for activity against developing tachyzoites of the $\mathrm{RH}$ strain of $T$. gondii in human fibroblast cell cultures.

Haloperidol, clozapine, and trifluoperazine were purchased from Sigma Chemical Company (St. Louis, Missouri). Fluphenazine, and thioridazine were gifts from E. R. Squib and Sons (Princeton, New Jersey), and Smith Kline Beecham (King of Prussia, Pennsylvania), respectively. Drugs were prepared as $1,000 \times$ stock solutions in dimethylsulfoxide (DMSO), and diluted into culture medium to the desired drug concentration and a final concentration of $0.1 \%$ DMSO. Both water and $0.1 \%$ DMSO controls were also tested.

Human foreskin fibroblast cells (Hs68, American Type Culture Collection, Manassas, Virginia) were maintained in RPMI 1640 medium (Lonza Walkersville, Inc., Walkersville, Maryland) supplemented with $10 \%$ fetal bovine serum (Atlanta Biologicals, Inc., Atlanta, Georgia), penicillin-streptomycin (100 U/ml-100 mg/ml each, Lonza Walkersville), and $1 \mathrm{mM}$ sodium pyruvate (Lonza Walkersville) were cultured in a

DOI: $10.1645 / G E-2536.1$ humidified, $37 \mathrm{C}$ incubator under a $5 \% \mathrm{CO}_{2}$ atmosphere. The $\mathrm{RH}$ strain (Sabin, 1941) of $T$. gondii was used for all experiments.

Tachyzoites were harvested from infected cell cultures as previously described (Strobl et al., 2009) and used immediately to infect monolayers of Hs68 cells in 48-well culture plates. Each culture well of Hs68 cells on the 48 -well culture plate was inoculated with $1 \times 10^{5}$ tachyzoites, and they were allowed to penetrate for $2-3 \mathrm{hr}$. Noninfecting tachyzoites were removed by a single medium exchange and $1 \mathrm{ml}$ of fresh medium composed of RPMI $1640+2 \%$ FBS and the test drugs or solvent control The cells were cultured for an additional $48 \mathrm{hr}$. The activity of each agent was determined using the SYBR green assay as previously described (Strobl et al., 2009). Briefly, the supernatant from the culture plates was centrifuged, aspirated, and the pellet was used to determine the proliferation of the tachyzoites. The DNA-binding fluorescent probe SYBR green (Molecular Probes, Carlsbad, California) was utilized at $1: 10,000$ dilution prepared in lysis buffer, containing $0.01 \%$ saponin and $0.1 \%$ Triton X-100, and added to the parasite pellets (Bennett et al., 2004). The pellets were incubated at $37 \mathrm{C}$ for $30 \mathrm{~min}$ to allow complete lysis of tachyzoites before being read on a fluorescent plate reader with excitation at $460 \mathrm{~nm}$ and emission at $530 \mathrm{~nm}$. The monolayers on the 48 -well plates were fixed and stained with the use of a $0.5 \%$ crystal violet-5\% formaldehyde- $50 \%$ ethanol- $0.85 \% \mathrm{NaCl}$ solution (Strobl et al., 2009) to compare to the results obtained using the SYBR green assay qualitatively and to compare monolayer destruction among treatments.

An additional experiment was conducted in triplicate in which tachyzoite production was measured as previously described (Strobl et al., 2007).

Statistical analyses were performed with the use of PrismV5.02 (GraphPad Inc, LaJolla, California). Triplicate determinations were averaged and concentration response curves prepared using a curve-fit to a $\log$ inhibitor-response model. Drug $\mathrm{IC}_{50}$ values were estimated from the curve-fits.

Clozapine and haloperidol did not have inhibitory activity in the assay when it was examined at 1,5 , or $10 \mu \mathrm{M}$; therefore, no $\mathrm{IC}_{50}$ could be calculated (data not shown). The mean and SEM from 2 independent experiments performed in triplicate was used to construct the graphs of activity for fluphenazine, thioridazine, and trifluoperazine (Fig. 1). Fluphenazine had an $\mathrm{IC}_{50}$ of $1.7 \pm 0.1 \mu \mathrm{M}$ and thioridazine had an $\mathrm{IC}_{50}$ of $1.2 \pm 0.1 \mu \mathrm{M}$. Trifluoperazine had slight stimulatory activity at lower levels $(2.0 \mu \mathrm{M})$ and inhibitory activity at higher levels $\left(\mathrm{IC}_{50} 3.8 \pm\right.$ $0.1 \mu \mathrm{M})$. Results observed in tachyzoite counting studies in 48-well plate studies were similar to those seen using the SYBR green assay (Fig. 2)

Jones-Brando et al. (2003) examined 12 neuroleptic agents against the RH strain of $T$. gondii growing in human fibroblast cells with the use of an ELISA-based assay to determine activity. They reported that the $\mathrm{IC}_{50}$ of haloperidol was $5.6 \mu \mathrm{g} / \mathrm{ml}(15 \mu \mathrm{M})$. Haloperidol was not active in the present study at any of the concentrations examined $(1-10 \mu \mathrm{M})$. We demonstrated activity of fluphenazine, thioridazine, and trifluoperazine against developing tachyzoites of $T$. gondii in the present study.

Schizophrenia usually occurs in women around the ages of 25 to $35 \mathrm{yr}$, which corresponds to peak reproductive years, leading to a use of these agents in pregnant women. Fortunately, no study has demonstrated an association between the use of antipsychotic agents during pregnancy and an increased risk for birth defects or other adverse outcomes (see Einarson and Boskovic, 2009). However, studies in humans with schizophrenia that were treated with the anti-T. gondii agents trimethoprim (Shibre et al., 2010) or azithromycin (Dickerson et al., 2009) failed to demonstrate significant improvement in clinical symptoms. Because antischizophrenia drugs are designed to act in the brain, it is likely that they may have more activity for the brain than other anti- $T$. gondii agents. Studies using rats 

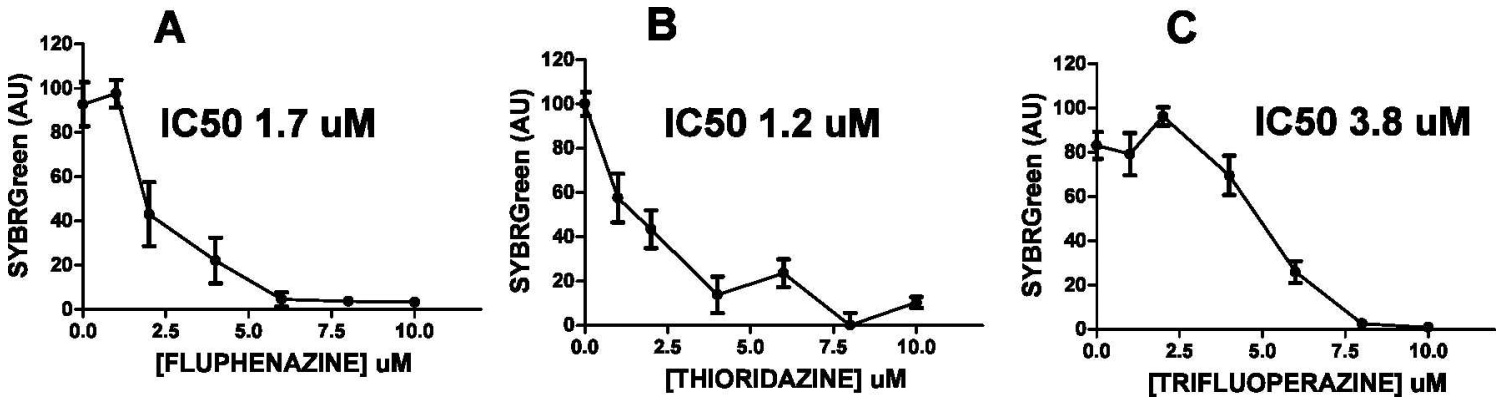

Figure 1. Activity of fluphenazine, thioridazine, and trifluoperazine against developing tachyzoites of Toxoplasma gondii in human fibroblast cell cultures using a SYBR green assay.

have reported that haloperidol or pyrimethamine plus dapsone are effective in ameliorating behavioral changes associated with chronic $T$. gondii infection (Webster et al., 2006). A study in mice using valproic acid did not demonstrate an effect on acute toxoplasmosis and there was no effect on $T$. gondii tissue cyst numbers in chronically infected mice (Goodwin et al., 2008).

Fluphenazine showed good activity against $T$. gondii in the present study (an $\mathrm{IC}_{50}$ of $1.7 \mu \mathrm{M}$ ). It is a promising drug for further investigation because fluphenazine has a highly acceptable adverse effect profile and is considered to be among the best tolerated anti-psychotic drugs available for patient use. Therapeutic plasma levels of fluphenazine are approximately $5 \mathrm{nM}$, well below the concentrations of drug that inhibited $T$. gondii proliferation in the present cell culture tests. Nevertheless, it is likely that levels of fluphenazine in the brain exceed those in plasma, and that fluphenazine could exert some anti- $T$. gondii actions within the central nervous system. The safety of fluphenazine for use in pregnant women has not been established (see Einarson and Boskovic, 2009); nevertheless, a case report showed a normal pregnancy and birth occurred in a woman who had taken fluphenazine continuously through her pregnancy (Cleary, 1977). In addition, there is a slow-release form of fluphenazine available that provides a convenient, sustained delivery of drug to enhance patient compliance. Thioridazine has also been administered to a pregnant woman without adverse effects to the baby (Yaris et al., 2004). We found thioridazine is slightly more potent than fluphenazine against $T$. gondii in cell culture, and because therapeutic plasma levels of thioridazine are in the range of $5 \mu \mathrm{M}$ (Kirchherr and Kuhn-Velten, 2006), drug levels in patients undergoing treatment with thioridazine are more than sufficient to exert an anti- $T$. gondii activity.

Several laboratories, including our own, have found that antipsychotic drugs and mood-stabilizing agents exert fairly potent effects on $T$. gondii

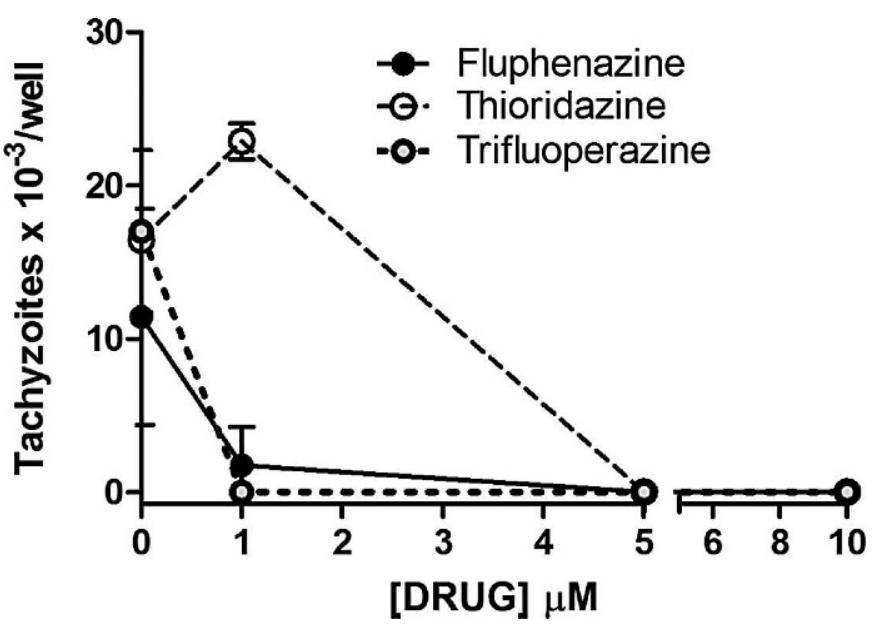

Figure 2. Activity of fluphenazine, thioridazine, and trifluoperazine against developing tachyzoites of Toxoplasma gondii in human fibroblast cell cultures using a tachyzoite counting assay. Data represent the percent of untreated control tachyzoite counts. tachyzoites (Pezzella et al., 1997; Jones-Brando et al., 2003; Strobl et al. 2007). However, defining a mechanism of action in $T$. gondii has proven to be more elusive. A confounding factor in elucidating the biological effects for these drugs in $T$. gondii is the existence of multiple pharmacological targets. For example, there are 2 reports that valproic acid, a drug approved for use as an antiepileptic and mood stabilizer, reduces tachyzoite proliferation (Jones-Brando et al., 2003; Strobl et al., 2007). The antiepileptic properties of valproic acid are generally attributed to its ability to raise levels of the neurotransmitter gamma-amino butyric acid (GABA) in the brain, as well as to inhibit certain sodium channels and Ttype calcium channels (Golan et al., 2008). There are also numerous reports that valproic acid is a histone deacetylase inhibitor and exerts broad biological activity by causing changes in protein acetylation (Monti et al., 2009). Deciphering which of these mechanisms drive the valproic acid response in tachyzoites is difficult and, though we have suggested that histone deacetylase is a key target of valproic acid in $T$. gondii based upon the antiproliferative actions of other chemical classes of histone deacetylase inhibitors in T. gondii (Strobl et al., 2007), there is no evidence that the regulation of ion channels, GABA metabolism, or other signaling pathways by valproic acid does not exert effects in $T$. gondii.

An equally complex picture emerges when examining the pharmacologic targets of the antipsychotic drugs. The typical antipsychotic drugs are chemically related and share a phenothiazine ring structure. This class of antagonists includes chlorpromazine, trifluoperazine, fluphenazine, and thioridazine, and their 2 primary pharmacological targets are the dopamine D2 receptor and calmodulin (Prozialeck and Weiss, 1982; Miyamoto et al., 2005). Haloperidol is a typical antipsychotic, a highly potent D2 dopamine receptor antagonist and lower-potency calmodulin inhibitor, but has a butyrophenone ring structure that is chemically distinct from the phenothiazine group. In addition to these main drug targets, chlorpromazine, trifluoperazine, fluphenazine, and haloperidol have been reported to inhibit several types of potassium channels, and to mediate intracellular calcium release through actions on the ryanodine receptor (Nakazawa et al., 1995; Suessbrich et al., 1997; Wagner et al., 2004; Qin et al., 2009). Second-generation antipsychotic drugs target the dopamine D2 receptor, as well as the serotonin receptor, HT-2A, and, as a class, bind less tightly to the dopamine receptor than typical antipsychotics (Seeman, 2002; Miyamoto et al., 2005). Clozapine is the prototype agent in this class, which also includes olanzapine, risperidone, and quetiapine (Miyamoto et al., 2005). Unlike the typical antipsychotics, the secondgeneration antipsychotics are not calmodulin antagonists, and even some cellular actions of clozapine show dependence upon calmodulin-activated kinase activity (Ninan et al., 2003; Shin et al., 2006). Of note, in our work, clozapine $(5 \mu \mathrm{M})$ was ineffective in protecting Hs68 host cell death through proliferation of tachyzoites and cell lysis; although an earlier publication showed that clozapine $\left(\mathrm{ID}_{50} 18 \mu \mathrm{M}\right.$ ) inhibited $T$. gondii replication in host cells, the same article showed the other secondgeneration antipsychotics tested, i.e., risperidone, quetiapine, and olanzapine, were inactive (Jones-Brando et al., 2003). We suggest that the differential response of $T$. gondii to typical and second generation antipsychotics implicates a role for calmodulin in the protection of $\mathrm{RH}$ $T$. gondii-infected $\mathrm{Hs} 68$ cells by trifluoperazine, fluphenazine, and thioridazine reported here. We recognize that there is no reason to exclude the idea that actions of these drugs on ion channels also contribute to inhibition of tachyzoite propagation in host cells. In fact, multiple mechanisms may be at work here. 
Transient rises in intracellular calcium within tachyzoites serve as a trigger for parasite egress from host cells and participate in the cell gliding motility and host-cell invasion processes critical to $T$. gondii survival (Hoff and Carruthers, 2002; Nagamune et al., 2008; Chandramohanadas et al., 2009; Lourido et al., 2010). Calcium binding activates calmodulin and downstream calmodulin-dependent protein kinases have been implicated in life-cycle and erythrocyte invasion of the apicomplexan, Plasmodium falciparum (Vaid et al., 2008; Wurtz et al., 2009), but these pathways are not well understood in $T$. gondii. In our experiments, tachyzoites were observed within parasitophorous vesicles in host cells suggesting that antipsychotics might blunt signals for $T$. gondii egress. This might involve calmodulin inhibition or, alternatively, the actions of antipsychotics on ion fluxes could act to repress the calcium signal for egress. Atypical antipsychotics stimulate excess calcium release from intracellular stores by opening ryanodine receptors (Wagner et al., 2004; Qin et al., 2009), and these drugs could thereby exert a direct cytotoxic effect on tachyzoites by amplification of the normal calcium release signals to initiate egress (Lovett and Sibley, 2003). Furthermore, there is good evidence that the actomyosin motor directing cell motility operates independently of calmodulin (HermGotz et al., 2002), yet calmodulin is nevertheless intimately associated with the actomyosin complex in the apical complex of the invading tachyzoite (Pezzella-D'Alessandro et al., 2001). Calmodulin may exert its role in invasion by mediating calcium-dependent conoid extrusion and through calcium-stimulated secretion of lytic enzymes at the adhesion site on the host cell membrane that serves to fluidize the host cell membrane and facilitate parasite entry. In these ways, calmodulin inhibition would suppress tachyzoite invasion and protect host cell monolayers.

The importance of dopamine receptor antagonism in the inhibition of T. gondii by antipsychotics is enigmatic. Clearly, the more potent D2 antagonists of the typical antipsychotic class are more active inhibitors of T. gondii than the less-potent second generation antipsychotics, but at this time, there is no good evidence for expression of $\mathrm{D} 2$ receptors on $T$. gondii. Fibroblast cells do not express detectable levels of dopamine receptors making it unlikely that the actions are indirectly mediated through altered biology of the host cell (Tang et al., 1994).

This work was supported by a grant from the Harvey W. Peters Research Foundation.

\section{LITERATURE CITED}

Alvarado-Esquivel, C., O. P. Alanis-Quiñones, M. Á. ArreolaValenzuela, A. Rodríguez-Briones, L. J. Piedra-Nevarez, E. Duran-Morales, S. Estrada-Martínez, S. A. Martínez-García, AND O. LiesENFELD. 2006. Seroepidemiology of Toxoplasma gondii infection in psychiatric inpatients in a northern Mexican city. BMC Infectious Diseases 6: 178 .

Bennett, T. N., M. Paguio, B. Gligorijevic, C. Seudieu, A. D. Kosar, E. Davidson, ANd P. D. Roepe. 2004. Novel, rapid, and inexpensive cellbased quantification of antimalarial drug efficacy. Antimicrobial Agents and Chemotherapy 48: 1807-1810.

Brown, S. A., C. A. Schaefer, C. P. Quesenberry, Jr., L. Liu, V. P. Babulas, and E. S. Susser. 2005. Maternal exposure to toxoplasmosis and risk of schizophrenia in adult offspring. American Journal of Psychiatry 162: 767-773.

CARTER, C. J. 2009. Schizophrenia susceptibility genes directly implicated in the life cycles of pathogens: Cytomegalovirus, influenza, Herpes simplex, rubella, and Toxoplasma gondii. Schizophrenia Bulletin 35: $1163-1182$.

Chandramohanadas, R., P. H. Davis, D. P. Beiting, M. B. Harbut, C. Darling, G. Velmourougane, M. Y. Lee, P. A. Greer, D. S. Roos, And D. C. Greenbaum. 2009. Apicomplexan parasites co-opt host calpains to facilitate their escape from infected cells. Science 324: 794-797.

Cetinkaya, Z., S. Yazar, O. Gecici, and M. N. Namli. 2007. AntiToxoplasma gondii antibodies in patients with schizophreniaPreliminary findings in a Turkish sample. Schizophrenia Bulletin 33: 789-791.

Cleary, M. F. 1977. Fluphenazine decanoate during pregnancy. American Journal of Psychology 134: 815-816.

Dickerson, F. B., J. J. Boronow, C. R. Stallings, A. E. Origoni, and R. H. YoLKEN. 2007. Toxoplasma gondii in individuals with schizophrenia: Association with clinical and demographic factors and with mortality. Schizophrenia Bulletin 33: 737-740.
, C. R. Stallings, J. J. Boronow, A. E. Origoni, and R. H. Yolken. 2009. A double-blind trial of adjunctive azithromycin in individuals with schizophrenia who are seropositive for Toxoplasma gondii. Schizophrenia Research 112: 198-199.

Einarson, A., AND R. BosKovic. 2009. Use and safety of antipsychotic drugs during pregnancy. Journal of Psychiatric Practice 15: 183-192.

Golan, D. E., A. H. Tashitan, JR., E. J. Armstrong, and A. W. Armstrong. 2008. Principles of pharmacology. The pathophysiologic basis of drug therapy, 2nd ed. Lippincott, Williams \& Williams, Philadelphia, Pennsylvania, 234 p.

Goodwin, D. G., J. Strobl, S. M. Mitchell, A. M. Zajac, and D. S. LiNDSAY. 2008. Evaluation of the mood-stabilizing agent valproic acid as a preventative for toxoplasmosis in mice and activity against tissue cysts in mice. Journal of Parasitology 94: 555-557.

Herm-Gotz, A., S. Weiss, R. Stratman, S. Fujita-Becker, C. Ruff, E. Meyhofer, T. Soldati, D. J. Manstein, M. A. Geeves, and D. SoldATI. 2002. Toxoplasma gondii myosin A and its light-chain: A fast, single-headed, plus-end-directed motor. The EMBO Journal 21: 2149-2158.

Hoff, E. F., And V. B. Carruthers. 2002. Is Toxoplasma egress the first step in invasion? Trends in Parasitology 18: 251-255.

Jones-Brando, L., E. F. Torrey, and R. Yolken. 2003. Drugs used in the treatment of schizophrenia and bipolar disorder inhibit the replication of Toxoplasma gondii. Schizophrenia Research 62: 237-244.

KirchHerR, H., AND W. N. Kunn-Velten. 2006. Quantitative determination of forty-eight antidepressant and antipsychotics in human plasma by HPLC tandem mass spectrometry: A multi-level sample approach. Journal of Chromatography B 843: 100-113.

Lourido, S., J. Shuman, C. Zhang, M. Shokat, R. Hiu, and L. D. Sibley. 2010. Calcium-dependent protein kinase 1 is an essential regulator of exocytosis in Toxoplasma. Nature 465: 359-362.

Lovett, J. L., And L. D. Sibley. 2003. Intracellular calcium stores in Toxoplasma gondii govern invasion of host cells. Journal of Cell Science 116: 3009-3016.

Miyamoto, S., G. E. Duncan, C. E. Marx, and J. A. Lieberman. 2005. Treatments for schizophrenia: A critical review of pharmacology and mechanisms of action of antipsychotic drugs. Molecular Psychiatry 10: $79-104$.

Mortensen, P. B., B. Nørgaard-Pedersen, B. L. Waltoft, T. L. Sørensen, D. Hougaard, and R. H. Yolken. 2007. Early infections of Toxoplasma gondii and the later development of schizophrenia. Schizophrenia Bulletin 33: 741-744.

-, E. F. TORREY, AND R. H. YoLKen. 2007. Toxoplasma gondii as a risk factor for early-onset schizophrenia: Analysis of filter paper blood samples obtained at birth. Biological Psychiatry 61: 688-693.

Monti, B., E. Polazzi, And A. Contestabile. 2009. Biochemical, molecular and epigenetic mechanisms of valproic acid neuroprotection. Current Molecular Pharmacology 2: 95-109.

Nagamune, K., L. M. Hicks, B. Fux, F. Brossier, E. N. Chini, and L. D. Sibley. 2008. Abscisic acid controls calcium-dependent egress and development in Toxoplasma gondii. Nature 45: 207-210.

Nakazawa, K., K. Ito, S. Koizumi, Y. Ohno, and K. Inoue. 1995 Characterization of inhibition by haloperidol and chlorpromazine of a voltage-activated $\mathrm{K}^{+}$current in rat phaeochromocytoma cells. British Journal of Pharmacology 116: 2603-2610.

Niebuhr, D. W., A. M. Millikan, D. N. Cowan, R. Yolken, Y. Li, And N. S. Weber. 2008. Selected infectious agents and risk of schizophrenia among U.S. military personnel. American Journal of Psychiatry 165: 99-106.

Ninan, I., K. E. Jardemark, X. Liang, and R. Y. Wang. 2003. Calciumcalmodulin-dependent kinase II is involved in the facilitating effect of clozapine on NMDA- and electrically evoked responses in the medial prefrontal cortical pyramidal cells. Synapse 47: 285-294.

Pezzella, N., A. Bouchot, A. Bonhomme, L. Pingret, C. Klein, H Burlet, G. Balosser, P. Bonhomme, and J. M. Pinon. 1997. Involvement of calcium and calmodulin in Toxoplasma gondii tachyzoite invasion. European Journal of Cell Biology 74: 92-101.

Pezzella-D'Alessandro, N., H. Le Moal, A. Bonhomme, A. Valero, C Klein, J. Gomez-Morino, and J. M. Pinon. 2001. Calmodulin distribution and the actomyosin cytoskeleton in Toxoplasma gondii. Journal of Histochemistry and Cytochemistry 49: 445-454. 
Prozialeck, W. C., AND B. Weiss. 1982. Inhibition of calmodulin by phenothiazines and related drugs: Structure activity relationships. Journal of Pharmacology and Experimental Therapeutics 222: 509-516.

Qin, J., A. V. Zima, M. Porta, L. A. Blatter, and M. Fill. 2009. Trifluoperazine a ryanodine receptor agaonist. Pflügers ArchivEuropean Journal of Physiology 458: 643-651.

Regier, D. A., W. E. Narrow, D. S. Rae, R. W. Manderscheid, B. Z. Locke, AND E. K. Goodwin. 1993. The de facto US mental and addictive disorders service system. Epidemiologic catchment area prospective 1-year prevalence rates of disorders and services. Archives of General Psychiatry 50: 85-94.

SABIN, A. B. 1941. Toxoplasmic encephalitis in children. Journal of the American Medical Association 116: 801-807.

Shibre, T., A. Alem, A. Abdulahi, M. Araya, T. Beyero, G. Medhin, N. Deyassa, A. Negash, A. Nigatu, D. Kebede, and A. Fekadu. 2010. Trimethoprim as adjuvant treatment in schizophrenia: A doubleblind, randomized, placebo-controlled clinical trial. Schizophrenia Bulletin 36: 846-851.

Shin, S. Y., B. H. Choi, J. Ko, S. H. Kim, Y. S. Kim, AND Y. H. Lee. 2006. Clozapine, a neuroleptic agent, inhibits Akt by counteracting $\mathrm{Ca}^{+2} /$ calmodulin in PTEN-negative U087MG human glioma cells. Cell Signaling 18: $1876-1886$.

Strobl, J. S., M. Cassell, S. M. Mitchell, C. M. Reilly, and D. S. LINDSAY. 2007. Scriptaid and suberoylanilidehydroxamic acid are histonedeacetylase inhibitors with potent anti-Toxoplasma gondii activity in vitro. Journal of Parasitology 93: 694-700.

, C. W. Seibert, Y. Li, R. Nagarkatti, S. M. Mitchell, A. C. Rosypal, D. Rathore, and D. S. Lindsay. 2009. Inhibition of Toxoplasma gondii and Plasmodium falciparum infections in vitro by NSC3852, a redox active antiproliferative and tumor cell differentiation agent. Journal of Parasitology 95: 215-223.

Suessbrich, H., R. Schonherr, S. H. Heinemann, B. Attali, F. Lang, AND A. E. Busch. 1997. The inhibitory effect of the antipsychotic drug haloperidol on HERG potassium channels expressed in Xenopus oocytes. British Journal of Pharmacology 120: 968-974.

Tang, L., R. D. Todd, A. Heller, and K. L. O’Malley. 1994 Pharmacological and functional characterization of D2, D2 and D4 dopamine receptors in fibroblast and dopaminergic cell lines. Journal of Pharmacology and Experimental Therapeutics. 268: 495502 .

Vaid, A., D. C. Thomas, and P. Sharma. 2008. Role of $\mathrm{Ca}^{+2} /$ calmodulinPfPKB signaling pathway in erythrocyte invasion by Plasmodium falciparum. Journal of Biological Chemistry 283: 5589-5597.

Wagner, R., R. H. Fink, and D. G. Stephenson. 2004. Effects of chlorpromazine on excitation-coupling events in fast-twitch skeletal muscle fibers of the rat. British Journal of Pharmacology 141: 624 633.

Wang, H. L., G. H. Wang, Q. Y. Li, C. Shu, M. S. Jiang, and Y. Guo. 2006. Prevalence of Toxoplasma infection in first-episode schizophrenia and comparison between Toxoplasma-seropositive and Toxoplas$m a$-seronegative schizophrenia. Acta Psychiatria Scandanavia 114: $40-48$.

Webster, J. P., P. H. Lamberton, C. A. Donnelly, and E. F. Torrey. 2006. Parasites as causative agents of human affective disorders? The impact of antipsychotic, mood-stabilizer and anti-parasite medication on Toxoplasma gondii's ability to alter host behaviour. Proceedings of the Royal Society B: Biological Science 273: 10231030

Wurtz, N., J. Desplans, and D. Parzy. 2009. Phenotypic and transcriptomic analysis of Plasmodium falciparum protein kinase A catalytic subunit inhibition. Parasitology Research 105: 16911699.

Yaris, F., E. Yaris, M. Kadioglu, C. Ulku, M. Kesim, and N. I. KALYONCU. 2004. Use of polypharmacotherapy in pregnancy: A prospective outcome in a case. Progress in Neuropsychopharmacology and Biological Psychiatry 28: 603-605. 